\title{
Variables personales relacionadas con el rendimiento académico
}

\author{
Gina Bojorque $^{1}$ (D), Vilma Bojorque ${ }^{2}$, Juana Dávalos ${ }^{1}$ \\ ${ }^{1}$ Facultad de Filosofía, Universidad de Cuenca, Cuenca, Ecuador. \\ ${ }^{2}$ Facultad de Ciencias Médicas, Universidad de Cuenca, Cuenca, Ecuador. \\ Autores para correspondencia: gina.bojorque@ucuenca.edu.ec, vilma.bojorque@ucuenca.edu.ec, \\ juana.davalos@ucuenca.edu.ec
}

Fecha de recepción: 3 de junio 2016 - Fecha de aceptación: 5 de septiembre 2016

\section{RESUMEN}

El objetivo del presente estudio fue examinar en qué medida variables de tipo personal como inteligencia, atención, autoconcepto y ansiedad predicen el rendimiento académico de los estudiantes de cuarto año de educación general básica. Un total de 410 niños procedentes de 14 escuelas públicas de la ciudad de Cuenca recibieron una serie de pruebas para evaluar su inteligencia (WISC-IV), atención (D2), auto-concepto (Piers-Harris), y ansiedad (Spence). Los resultados demuestran en primer lugar, que los niveles de autoconcepto y ansiedad promedio de los niños fue moderado, sin embargo, su nivel medio de inteligencia fue normal-bajo y su nivel de atención fue extremadamente bajo. En segundo lugar, se encontró que todas las variables personales mencionadas predicen el rendimiento académico, siendo la inteligencia la variable que presenta el índice más alto de predicción. Finalmente, se encontraron diferencias de género en todas las variables, indicando que las mujeres obtuvieron puntuaciones más altas en inteligencia, atención y autoconcepto, así como un nivel de ansiedad mayor. Las niñas también presentaron un rendimiento académico mayor. Los resultados de este estudio apuntan a la necesidad de llevar a cabo intervenciones educativas orientadas a estimular el desarrollo de variables personales, incluyendo la inteligencia, con el fin de favorecer el desempeño académico de nuestros estudiantes, particularmente de aquellos en riesgo de fracaso escolar.

Palabras clave: Rendimiento académico, inteligencia, atención, autoconcepto, ansiedad.

\begin{abstract}
The present study examined to what extend some personal variables such as intelligence, attention, selfconcept, and anxiety, predict four-grade children's academic achievement. A total of 410 children form 14 public schools of the city of Cuenca were subjected to a series of tests focusing on intelligence (WISC), attention (D2), self-concept (Piers-Harris), and anxiety (Spence). Results revealed that children's levels of self-concept and anxiety were normal; however, their level of intelligence was normal-low and their level of attention was extremely low. Second, it was found that all the mentioned variables predicted children's academic achievement, with intelligence possessing the highest predicting validity. Finally, gender differences were consistently found in all the variables, indicating that girls obtained higher scores of intelligence, attention, and self-concept, as well as higher levels of anxiety. The results of this study point to the need of implementing educational interventions aimed at stimulating the development of personal variables, including intelligence, to increase children's academic achievement, particularly for those students at risk of academic failure.
\end{abstract}

Keywords: School achievement, intelligence, attention, self-concept, anxiety. 


\section{INTRODUCCIÓN}

Durante los últimos años se han realizado varios estudios encaminados a determinar cuáles son los factores que influyen en el rendimiento académico de niños y jóvenes (Berg, 2008; Castillo-Parra et al., 2009; Spinath et al., 2010) con miras a realizar intervenciones que prevengan el fracaso escolar. TentiFanfani (2002) señala que el rendimiento académico no es producto de una causa única, sino que existen diversas variables que inciden en el mismo tales como habilidades, expectativas de logro, rasgos de personalidad, factores genéticos, socioeconómicos, culturales, educativos, entre otros. De acuerdo a Gonzalez-Pienda (2003) estas variables del rendimiento académico se agrupan en dos niveles: personales y contextuales. Entre las variables de tipo personal se encuentran, por ejemplo, la inteligencia, la memoria, la atención, el autoconcepto, las emociones, y la ansiedad. Las variables contextuales pueden ser socio-ambientales (p.e., familia, nivel socio-económico, lugar de residencia), institucionales (p.e., tipo de centro educativo, formación de profesores, clima académico) e instruccionales (p.e., contenidos, métodos de enseñanza, uso de tecnologías). Sin embargo, resulta evidente que algunas variables contextuales como factores demográficos, nivel socio-económico, escolaridad de los padres, entre otras resultan difíciles de ser afectadas cuando se pretenden diseñar programas de intervención que ayuden a prevenir o mejorar el fracaso académico; por ello, es importante contar con datos de variables de índole personal que permitan implementar programas preventivos (Caso-Niebla \& Hernández-Guzmán, 2007).

Diversas investigaciones encaminadas al estudio del rendimiento académico han asociado el mismo con una o más de las variables personales arriba mencionadas (Fernández-Castillo \& Gutiérrez, 2009; Miñano \& Castejón, 2008; Spinath et al., 2010). Por ejemplo, Miñano \& Castejón (2008) reportaron que la inteligencia general, el autoconcepto, las metas y las estrategias de aprendizaje contribuyen al rendimiento académico, siendo la inteligencia la variable con mayor poder predictivo. Por su parte, Fernández-Castillo \& Gutiérrez (2009) encontraron que la ansiedad y la depresión predicen el rendimiento académico global y que la atención selectiva se asocia únicamente con el rendimiento académico en matemáticas. Entre las variables de tipo personal que más se relacionan con el rendimiento académico se encuentran la inteligencia, la atención, el auto-concepto y la ansiedad (García-Fernández et al., 2013; Gottfredson, 2002; León, 2008; Loperena, 2008).

La inteligencia es un conjunto de capacidades o habilidades mentales de selección y comprensión de información para adaptar los conocimientos y experiencias a situaciones cotidianas (Fandiño, 2008); siendo esta la manera cómo el individuo organiza su experiencia con el medio ambiente físico y social (Gareca, 2005). La inteligencia general ha sido determinada por varios autores como el predictor más fuerte del rendimiento académico (Cascón, 2000; Gottfredson, 2002; Spinath et al., 2010). Por ejemplo, Cascón (2000) señala que la inteligencia es el factor psicopedagógico que mejor predice el rendimiento académico y que mediante el empleo oportuno de pruebas de inteligencia estandarizadas es posible detectar grupos en riesgo de evidenciar fracaso escolar. De la misma manera, en un estudio transversal llevado a cabo en Estonia, con una muestra de 3618 estudiantes de educación básica, se encontró que el nivel de inteligencia de los estudiantes fue el mejor predictor de sus calificaciones en todos los grados que participaron en la investigación (Laidra et al., 2007).

La atención, definida como un mecanismo que activa el funcionamiento de los procesos de selección, distribución y mantenimiento de la actividad psicológica (García-Sevilla, 1997), es otra variable de tipo personal que la literatura asocia con el rendimiento académico (Castillo-Parra et al., 2009; León, 2008). León (2008) señala que las medidas de atención han sido típicamente consideradas como predictores del rendimiento académico y que los ejercicios de atención plena reportan grandes beneficios para el aprendizaje. Por el contario, al presentarse dificultad en los procesos antes descritos, el procesamiento de la información se ve disminuido y como consecuencia también el aprendizaje y el rendimiento académico (Fernández-Castillo \& Gutiérrez, 2009; León, 2008).

El autoconcepto hace referencia a la forma en que las personas se perciben o ven a sí mismas (Milicic, 2001); ello es el resultado de un proceso de análisis, valoración e integración de la información que proviene de su experiencia y de la retroalimentación de los demás (Naranjo \& González, 2012). El autoconcepto es otra variable que explica el rendimiento académico de los niños (Loperena, 2008; Sánchez \& Peralta, 2003), pues tiene relación con la forma en que el alumno enfrenta los problemas y 
el trabajo académico, así como con el tipo de relaciones que establece con los docentes y compañeros (Arancibia, 1990; citado en Villarroel, 2001). Así, en un estudio llevado a cabo con 245 alumnos de primaria en España, se reportó que existe una estrecha relación entre las medidas de autoconcepto y el rendimiento académico de los estudiantes (Sánchez \& Peralta, 2003). Los niños que presentan niveles de rendimiento superiores, generalmente suelen verse a sí mismos como altamente competentes, demuestran una actitud positiva ante el trabajo escolar, están satisfechos con su desempeño, todo lo cual hace que su interés por aprender aumente.

La ansiedad es otra variable relacionada con el rendimiento académico en virtud de sus repercusiones en el aprendizaje. Se podría decir que la ansiedad es un estado de miedo o inquietud ante situaciones desagradables y el intento frecuente de evitarlas. Por ejemplo, los estudiantes que presentan ansiedad hacia las matemáticas demuestran una experiencia extrema de malestar al realizar tareas matemáticas o incluso simplemente pensar en ellas, se ponen nerviosos, reducen su motivación y tratan de evitar tareas relacionadas con esta materia (García et al., 2016). Un nivel moderado de ansiedad ayuda a enfrentar situaciones de mejor manera pues, se genera en situaciones de estrés que producen en la persona un nivel de alarma que mejoraría su rendimiento en cualquier tarea que lo requiera (Victor \& Rooper, 2002). En este sentido, se encontró que los estudiantes con alto rendimiento en las áreas de lengua y matemáticas, presentaron puntuaciones de ansiedad significativamente más altas ante el fracaso académico y la agresión en comparación con los estudiantes que obtuvieron un bajo rendimiento en estas áreas (García-Fernández et al., 2013). Sin embargo, los niveles de ansiedad demasiado elevados reducen la eficiencia en el aprendizaje, ya que disminuyen la atención, la concentración y el esfuerzo sostenido, con la consecuente disminución del rendimiento en cualquier tarea (Rains, 2004; Mazzone et al., 2007).

Por otro lado, diversos estudios han puesto en evidencia que existen diferencias de género en el desempeño de los estudiantes en las variables arriba mencionadas (Geary, 1996; Caso-Niebla \& Hernández-Guzmán, 2007; Hyde \& Linn, 1988; Locker \& Cropley, 2004; Tejedor, González-Gonzáles \& García-Senoran, 2008) siendo las mujeres las que presentan puntajes más altos en la mayoría de las medidas. Así, en un estudio llevado a cabo por Caso-Niebla \& Hernández-Guzmán (2007) se encontró que las mujeres presentan mejor rendimiento académico, motivación y habilidades de estudio, pero una autoestima más baja que los hombres. Los resultados de las pruebas TERCE ${ }^{1}$ (2016) indicaron una ventaja significativa de las niñas en el área de lectura y escritura frente a los niños, pero en el área de matemáticas se observó una ventaja similar de los niños frente a las niñas. En cuanto a diferencias de género en la capacidad intelectual, se encontró que los niños se desempeñan mejor que las niñas en pruebas de representación espacial de relaciones matemáticas, probablemente por su ventaja en habilidades espaciales (Geary, 1996), mientras que las niñas se desempeñan mejor en pruebas de habilidad verbal (Hyde \& Linn, 1988). Por su parte, Tejedor et al. (2008) encontraron diferencias de género en el empleo de estrategias de atención, siendo mayor la frecuencia de uso en las mujeres que en los hombres. De igual forma, se reportó que las mujeres muestran mayores niveles de ansiedad que los hombres inmediatamente antes de los exámenes (Locker \& Cropley, 2004).

Por lo expuesto, detectar las variables relacionadas con el rendimiento académico con la finalidad de intervenir en las mismas es fundamental para ayudar a los estudiantes a desempeñarse bien en sus estudios. Las consecuencias de un bajo rendimiento académico generan dificultades de índole personal y social que pueden comprometer el porvenir de los estudiantes (Gonzalez-Pienda, 2003). Desafortunadamente, tanto a nivel nacional (INEVAL ${ }^{2}$, 2014) como internacional (TERCE, 2015) el rendimiento académico de los niños ecuatorianos es bajo. A pesar de su importancia, investigaciones sobre las variables personales asociadas al rendimiento académico a nivel local son escasas. Por ello, el objetivo del presente estudio es examinar en qué medida las variables de tipo personal como inteligencia, atención, autoconcepto, ansiedad, predicen el rendimiento académico de los estudiantes de cuarto año de educación general básica de la ciudad de Cuenca. Nuestros hallazgos permitirán sentar las bases sobre las cuales se fundamenten futuros estudios sobre el tema, así como generar políticas

1 TERCE: Tercer Estudio Regional Comparativo y Explicativo: Ecuador mejora sus resultados 2006-2013. Disponible en http://www.evaluacion.gob.ec/resultados/images/_in2_bin/DAGI_

Terce14_InformeEjecutivo_20141203.pdf, 56 pp.

2 INEVAL: Instituto Nacional de Evaluación Educativa (http://www.evaluacion.gob.ec) 
educativas que apunten a mejorar el rendimiento académico de los niños y por lo tanto a mejorar su calidad de vida.

Las preguntas de investigación de este estudio son:

1. ¿En qué medida la inteligencia, la atención, el autoconcepto y la ansiedad predicen el rendimiento académico de los niños de cuarto año de básica?

2. ¿Existen diferencias significativas entre niños y niñas en su rendimiento escolar, inteligencia, atención, autoconcepto y ansiedad?

\section{MÉTODO}

\subsection{Participantes}

Los participantes fueron 410 niños de cuarto año de educación general básica (189 hombres, 221 mujeres) procedentes de 14 instituciones educativas públicas urbanas de la ciudad de Cuenca. La muestra incluyó a todos los niños matriculados que asistían normalmente a clases. El 73\% de niños ecuatorianos asisten a escuelas públicas; de este porcentaje, 39\% lo hacen dentro del área urbana, y $34 \%$ en el área rural (Ministerio de Educación, 2013). La edad media de los participantes al momento de la aplicación del test fue de 8 años, 2 meses (DT = 6.8 meses).

\subsection{Materiales}

Escala de inteligencia de Wechsler para niños -IV (WISC-IV)

La capacidad de inteligencia de los niños fue evaluada mediante la escala de inteligencia de Wechsler para niños -IV (WISC-IV) (Wechsler, 2005). Este test evalúa la capacidad cognitiva global y cuatro dominios específicos de inteligencia que son: (1) Comprensión verbal, a través de las siguientes subescalas: semejanzas, vocabulario, comprensión, información, adivinanzas; (2) Razonamiento perceptivo, por medio de las siguientes subescalas: cubos, conceptos, matrices y figuras incompletas; (3) Memoria de trabajo, mediante las siguientes subescalas: dígitos, letras y números, aritmética; y (4) Velocidad de procesamiento, a través de las siguientes subescalas: claves, búsqueda de símbolos, animales. La administración de este test es individual y toma entre 60 y 110 minutos por niño. El índice de confiabilidad del WISC-IV es de .97.

\section{Test de atención D2}

Para evaluar la capacidad de atención de los niños, se empleó el test de atención D2 (Brickenkamp, 2002). El D2 evalúa la atención selectiva mediante una tarea de cancelación. Mide la velocidad de procesamiento, el seguimiento de unas instrucciones y la precisión/eficiencia de la ejecución en una tarea de discriminación de estímulos visuales similares. El test ofrece nueve puntuaciones distintas que informan acerca de la velocidad y la precisión junto con otros aspectos importantes como son la estabilidad, la fatiga y la eficacia de la inhibición atencional. La aplicación del test es de forma individual o colectiva y toma alrededor de 10 minutos. Los niveles de confiabilidad del D2 son generalmente altos, reportándose coeficientes Alfa de Cronbach de hasta .98 (Culbertson \& Zillmer, 1998).

\section{Escala de autoconcepto de Piers-Harris}

Para evaluar el autoconcepto de los niños se empleó la Escala de Autoconcepto de Piers-Harris (Piers $\&$ Harris, 1969). Este test está diseñado para obtener información sobre la percepción que el alumno tiene de sí mismo (autoconcepto global) y de cómo valora diferentes aspectos de su forma de ser y su comportamiento. Evalúa las siguientes dimensiones: comportamiento, estatus académico, apariencia, ansiedad, popularidad y satisfacción. La escala consta de 80 frases sencillas con respuesta dicotómica (Sí/No). Su administración puede ser individual o colectiva, requiriendo alrededor de 30 minutos para completarlo. El índice de confiablidad de este test es de .89 . 


\section{Escala de ansiedad para niños de Spence $\left(\mathrm{SCAS}^{3}\right)$}

El nivel de ansiedad de los niños fue evaluado mediante la escala de ansiedad para niños de Spence (SCAS) (Henández-Guamán et al., 2009). Esta prueba está compuesta por 38 ítems referidos a síntomas de ansiedad con cuatro opciones tipo Likert: nunca (0), a veces (1), muchas veces (2) y siempre (3). Incluye, además, 6 ítems positivos de relleno para contrarrestar el sesgo negativo de los anteriores, y cuya calificación no se califica, ni se toma en cuenta en los análisis. Se califica mediante la suma de los puntos obtenidos para cada ítem, a mayor puntaje, mayor el nivel de ansiedad. Este test se puede aplicar individual o colectivamente. Su aplicación toma entre 25 y 30 minutos y generalmente presenta un índice de confiabilidad de 0.93 .

\section{$\underline{\text { Rendimiento académico }}$}

El rendimiento académico se determinó mediante el promedio de calificaciones escolares alcanzado por los niños al término del primer quimestre, pues según Lambating \& Allen (2002) estas constituyen el medio más empleado para evaluar el cumplimiento de los objetivos de aprendizaje dentro de las aulas de clases.

\section{Análisis de datos}

Para describir el rendimiento académico, inteligencia, atención, autoconcepto, y ansiedad se calcularon las medias, desviaciones típicas y rangos de los puntajes alcanzados por los niños en cada variable. Para analizar si la inteligencia, la atención, el autoconcepto y la ansiedad predicen el rendimiento académico, se realizó un análisis correlacional y de regresión lineal múltiple. Finalmente se condujo un análisis de varianza (ANOVA) para determinar si existen diferencias significativas entre niños y niñas en su rendimiento académico, inteligencia, atención, autoconcepto y ansiedad.

\subsection{Procedimiento}

Antes de administrar los tests, se obtuvo el consentimiento informado de los padres de familia de cada uno de los niños. Los test fueron aplicados durante los primeros dos meses del año lectivo, durante las jornadas de clases. El test de inteligencia (WISC-IV) se llevó a cabo de manera individual, en un aula separada y libre de ruidos. Los tests de atención (D2), autoconcepto (Piers-Harris) y ansiedad (Spence) fueron aplicados de manera colectiva en el salón de clases de los estudiantes. El nivel de rendimiento académico de los niños fue solicitado a cada una de las instituciones educativas.

Los tests fueron administrados por 6 evaluadoras entrenadas previamente para su aplicación. Los tests fueron calificados por un miembro del equipo de investigación con experiencia en la administración y calificación de los mismos, siguiendo las instrucciones presentadas en los manuales de cada uno.

\section{RESULTADOS}

Los resultados se presentan en dos partes. En primer lugar, se presentan los resultados descriptivos de cada una de las variables personales, así como del rendimiento académico de los niños. A continuación, se abordan las preguntas de investigación.

\subsection{Análisis descriptivo}

En la Tabla 1 se presentan los estadísticos descriptivos de los puntajes del rendimiento académico, inteligencia, atención, autoconcepto, y ansiedad. Estos datos indican en primer lugar que el rendimiento académico de los niños se encuentra en un nivel medio, existiendo grandes diferencias individuales, con niños que han alcanzado el rendimiento mínimo requerido para aprobar el año de 7/10 mientras que

${ }^{3}$ SCAS: Spence Children's Anxiety Scale 
otros han alcanzado rendimientos altos de hasta 9.89/10. En segundo lugar, el nivel de inteligencia promedio de los niños participantes corresponde a la categoría de normal bajo, existiendo grandes diferencias individuales entre niños que se encuentran por debajo de un nivel de inteligencia básico y aquellos con un nivel muy superior. En tercer lugar, el nivel promedio de atención es bajo y existen grandes diferencias entre los niños pues algunos reportan niveles de atención excesivamente bajos en tanto que otros han alcanzado niveles altos. En cuarto lugar, el nivel promedio de autoconcepto es normal alto, con niños que presentan niveles bajos de autoconcepto y otros que presentan niveles bastante altos. Finalmente, se observa un nivel moderado de ansiedad, existiendo igualmente grandes diferencias individuales, con niños que presentan niveles severos de ansiedad mientras que otros presentan niveles leves de ansiedad.

Tabla 1. Medias, desviaciones típicas y rangos de los puntajes del rendimiento académico, inteligencia, atención, autoconcepto, y ansiedad.

\begin{tabular}{lccc}
\hline \multicolumn{1}{c}{ Variable } & Media & Desviación típica & Rango \\
\hline Rendimiento académico & 8.75 & 0.61 & $7.01-9.89$ \\
Inteligencia & 86.90 & 16.02 & $29-156$ \\
Atención & 52.88 & 45.45 & $0-215$ \\
Autoconcepto & 58.85 & 10.56 & $23-76$ \\
Ansiedad & 33.11 & 17.47 & $1-85$ \\
\hline
\end{tabular}

\subsection{Preguntas de investigación}

Para responder la primera pregunta de investigación: ¿En qué medida la inteligencia, la atención, el autoconcepto y la ansiedad predicen el rendimiento académico de los niños de cuarto año de básica? en primer lugar se realizó un análisis correlacional. En vista de que ninguno de los datos de los cuatro tests siguen una distribución normal, se calculó la correlación de Spearman (Pedhazur, 1997) entre los puntajes alcanzados por los niños en el rendimiento académico, inteligencia, atención, autoconcepto y ansiedad. Los resultados de este análisis se reportan en la Tabla 2.

Como se puede apreciar, se encontró una correlación positiva entre el rendimiento académico y cada una de las cuatro variables personales, lo que sugiere que los niños que presentan niveles de inteligencia, atención, autoconcepto y ansiedad altos, también presentan un rendimiento académico alto. Los resultados de la Tabla 2 revelan que la inteligencia es la variable que más altamente se correlaciona con el rendimiento académico $(\rho=.40)$. Adicionalmente se encontró que existe una correlación positiva entre tres variables: inteligencia, atención y autoconcepto. La ansiedad solo se correlaciona con autoconcepto, siendo esta correlación inversamente proporcional, es decir que mientras menor es el nivel de ansiedad, mayor es el nivel de autoconcepto.

Tabla 2. Correlaciones de Spearman entre rendimiento académico, inteligencia, atención, autoconcepto y ansiedad.

\begin{tabular}{lccccc}
\hline & Rendimiento & Inteligencia & Atención & Autoconcepto & Ansiedad \\
\hline Rendimiento & 1 & $.40^{* *}$ & $.35^{* *}$ & $.33^{* *}$ & $.14^{*}$ \\
Inteligencia & $.40^{* *}$ & 1 & $.38^{* *}$ & $.34^{* *}$ & .06 \\
Atención & $.35^{* *}$ & $.38^{* *}$ & 1 & $.26^{* *}$ & .03 \\
Autoconcepto & $.34^{* *}$ & $.34^{* *}$ & $.26^{* *}$ & 1 & $-.22^{* *}$ \\
Ansiedad & $.14^{*}$ & .06 & .03 & $-.22^{* *}$ & 1 \\
\hline
\end{tabular}

** $p=0.01 ; * p=0.05$

En vista de las correlaciones significativas, a continuación, se llevó a cabo un análisis de regresión lineal múltiple para examinar si, y hasta qué punto, la inteligencia, la atención, el autoconcepto y la ansiedad predicen el rendimiento académico de los niños de cuarto año de básica. Para ello se corrieron cuatro modelos: (a) inteligencia (Modelo 1); (b) inteligencia + atención (Modelo 2); (c) inteligencia + atención + autoconcepto (Modelo 3); y (d) inteligencia + atención + autoconcepto + ansiedad (Modelo 4). En la Tabla 3 se presentan los resultados de este análisis por modelo. 
Tabla 3. Modelos de regresión lineal para predecir el rendimiento académico de los niños.

\begin{tabular}{clccccc}
\hline Modelo & Predictor & $\mathrm{F}$ & $\mathrm{B}$ & Error estándar & $\beta$ & $\sigma$ \\
\hline 1 & Inteligencia & $89.652^{*}$ & .016 & .002 & .424 & .000 \\
\hline 2 & 1_Inteligencia & $57.996^{*}$ & .013 & .002 & .346 & .000 \\
& 2_Atención & & .003 & .001 & .218 & .000 \\
\hline 3 & 1_Inteligencia & $45.100^{*}$ & .011 & .002 & .296 & .000 \\
& 2_Atención & & .003 & .001 & .195 & .000 \\
& 3_Autoconcepto & & .010 & .003 & .179 & .000 \\
\hline 4 & 1_Inteligencia & $37.525^{*}$ & .011 & .002 & .280 & .000 \\
& 2_Atención & & .003 & .001 & .188 & .000 \\
& 3_Autoconcepto & & .013 & .003 & .222 & .000 \\
& 4_Ansiedad & & .005 & .002 & .149 & .001 \\
\hline
\end{tabular}

$\mathrm{R}^{2}=.18\left(\right.$ Modelo 1); $\mathrm{R}^{2}=.22($ Modelo 2$) ; \mathrm{R}^{2}=.24($ Modelo 3$) ; \mathrm{R}^{2}=.26($ Modelo 4$) * p=<.001$

Como se resume en la Tabla 3, los resultados del análisis de regresión demuestran en primer lugar que la inteligencia estuvo predictivamente relacionada con el rendimiento académico. Más específicamente, el $18 \%$ de la varianza en el rendimiento académico de los estudiantes puede ser atribuida a su nivel de inteligencia. En segundo lugar, al añadir la atención como predictor en el análisis de regresión (Modelo 2) se observó un incremento del 4\% de la varianza explicativa en el rendimiento académico, lo que significa que la atención predice, aunque en menor medida, el rendimiento académico de los estudiantes aun cuando se controla estadísticamente el nivel de inteligencia. En tercer lugar, cuando se añadió en el análisis el autoconcepto como tercer factor de predicción (Modelo 3), el aumento de la varianza explicativa del rendimiento académico fue igualmente del $2 \%$, lo que quiere decir que el autoconcepto también es una variable productora del rendimiento académico aun cuando los niveles de inteligencia y atención sean estadísticamente controlados. Finalmente, al añadir la ansiedad como cuarto factor predictivo del rendimiento académico se encontró que la ansiedad explicó un $2 \%$ adicional de la varianza en el rendimiento académico de los estudiantes, luego de controlar estadísticamente la inteligencia, atención y autoconcepto. Estos resultados junto con los valores estandarizados $\beta$ (Tabla 3) indican que la inteligencia es el predictor más fuerte del rendimiento académico en comparación con la atención, autoconcepto y ansiedad.

En conjunto, los resultados del análisis de regresión indican que los niños con un nivel de inteligencia alto, y en menor medida con niveles de atención, autoconcepto y ansiedad también altos tienden a desempeñarse académicamente mejor.

Para responder a la segunda pregunta de investigación: ¿Existen diferencias significativas entre niños y niñas en su rendimiento escolar, inteligencia, atención, autoconcepto y ansiedad? se realizó un ANOVA de un factor, tomando a la variable género como variable de agrupamiento y a las medidas de rendimiento escolar, inteligencia, atención, autoconcepto y ansiedad como variables dependientes. Los resultados demuestran que existen diferencias significativas tanto en rendimiento escolar $F(1,408)=$ 9.436, $p=.002$, como en inteligencia $F(1,408)=7.826, p=.005$, atención $F(1,408)=5.693, p=.017$, autoconcepto $F(1,408)=7.302, p=.007$, y ansiedad $F(1,408)=3.796, p=.052$, siendo las niñas las que presentan los niveles más altos en todas las variables mencionadas.

\section{DISCUSIÓN}

El rendimiento académico de los estudiantes ecuatorianos tanto en pruebas nacionales como internacionales es bajo, lo cual es una situación preocupante ya que el éxito académico durante la escolaridad juega un papel importante en las oportunidades que los estudiantes van a tener en el futuro (Laidra et al., 2007). Estudios previos han reportado que la inteligencia, la atención, el autoconcepto y la ansiedad se relacionan con el rendimiento académico de los niños (García-Fernández et al., 2013; Gottfredson, 2002; León, 2008; Loperena, 2008); sin embargo, estos estudios han sido llevados a cabo en otros países, siendo escasas a nivel nacional. Por ello, el objetivo del presente estudio fue examinar 
en qué medida ciertas variables de tipo personal como la inteligencia, la atención, el autoconcepto y la ansiedad predicen el rendimiento académico de los estudiantes de cuarto año de básica de la ciudad de Cuenca. Para ello se aplicó a los estudiantes un conjunto de pruebas para evaluar su inteligencia (WISCIV), autoconcepto (Piers-Harris), ansiedad (Spence) y atención (D2). Este trabajo constituye un aporte local ya que pone a la luz nuevos e importantes conocimientos sobre las variables personales que influyen en el aprendizaje de los estudiantes cuencanos. El conocimiento del comportamiento y la interacción entre estas variables y el rendimiento académico permitirán fundamentar el diseño e implementación de programas que ayuden a mejorar el rendimiento académico de los estudiantes ecuatorianos.

Los resultados de este estudio demuestran, en primer lugar, que el rendimiento académico de los niños fue medio, lo cual no es consistente con reportes nacionales (INEVAL, 2014) e internacionales (TERCE, 2015) en los cuales se evidencia un nivel de rendimiento bajo en las pruebas aplicadas. Una posible explicación a este hecho es que, en el presente estudio se reportó el rendimiento académico global de los estudiantes, mientras que en las pruebas nacionales e internacionales arriba mencionadas se reportó el rendimiento académico por las áreas de estudio, es decir, lengua, matemáticas, ciencias naturales y estudios sociales, sin incluir otras áreas como cultura física, computación, arte, entre otras. Tal vez, estas últimas áreas son las que contribuyen a que el rendimiento académico global de los estudiantes sea mayor. Por otro lado, también es posible que las pruebas tomadas en las instituciones educativas, para evaluar el rendimiento académico de los estudiantes, sean menos exigentes que las pruebas tomadas en las evaluaciones nacionales e internacionales, siendo esta es una hipótesis que debería comprobarse en estudios futuros.

En segundo lugar, el desempeño promedio de los niños en las medidas de autoconcepto y ansiedad fue moderado, sin embargo, sorpresivamente, el promedio en las mediadas de inteligencia y atención de los niños fue bajo. Lo último difiere de resultados de estudios previos en los que se reportaron promedios mayores tanto inteligencia como de atención de los niños participantes (Jiménez et al., 2007). Estos resultados apuntan a la necesidad de realizar esfuerzos por aumentar los niveles de inteligencia y atención de los estudiantes del país pues estas variables son importantes no solo para el rendimiento académico sino también para desempeñarse adecuadamente en los distintos ámbitos de la vida.

En tercer lugar, consistente con estudios anteriores (García-Fernández et al., 2013; Gottfredson, 2002; León, 2008; Loperena, 2008), se encontró que la inteligencia la atención, el autoconcepto y la ansiedad predicen el rendimiento académico de los niños. Más aún, en combinación, estas cuatro variables representan el 26\% de la varianza total del rendimiento académico. Como era de esperarse, en base a estudios previos (Gottfredson, 2002; Laidra et al., 2007; Spinath et al., 2010), la inteligencia, fue el mayor predictor del rendimiento académico de los niños. Un análisis más detallado de estos resultados nos lleva a señalar que los niños que se desempeñan académicamente mejor en la escuela son aquellos que tienen mayor capacidad cognitiva global, son más veloces en procesar y seguir instrucciones, así como más capaces de discriminar estímulos visuales; presentan en mayor grado actitudes de respeto por sí mismos, y muestran un nivel medio de ansiedad. El hallazgo encontrado que señala a la inteligencia como el mayor predictor del rendimiento académico apunta a la necesidad de contar con programas de intervención que ayuden a mejorar las habilidades intelectuales de los estudiantes. Por ejemplo, la implementación de $\mathrm{PIENSO}^{4}$ (Yuste et al., 2009) ampliamente conocido y extendido en varios centros escolares en España podría ayudar a mejorar las habilidades intelectuales de los niños y por ende contribuir al mejoramiento de su rendimiento académico.

Finalmente, con respecto a las diferencias de género, consistente con estudios previos, las mujeres presentaron un mejor rendimiento académico que los hombres (Caso-Niebla \& Hernández-Guzmán, 2007; Laidra et al., 2007), así como niveles más altos de inteligencia, atención, autoconcepto, y ansiedad (Hyde \& Linn, 1988; Locker \& Cropley, 2004; Tejedor et al., 2008). Aunque estudios anteriores reportaron que los hombres se desempeñan mejor en el área de matemáticas y demuestran mayor habilidad espacial que las mujeres (Geary, 1996; TERCE, 2016), nuestra investigación no pudo corroborar estos resultados ya que no se obtuvo información detallada en ninguna de las variables estudiadas (ver abajo limitaciones de este estudio). Coincidimos con las recomendaciones realizadas en

4 PIENSO: Programa Integral de Estimulación de la Inteligencia 
TERCE (2016, pp. 1), que para superar las inequidades educativas significativas por género en logros de aprendizajes "es indispensable revisar el contenido del currículum y los materiales educativos, de forma que retraten de manera equitativa a hombres y mujeres en actividades de distinta índole".

Todos estos hallazgos, sumados a las grandes diferencias individuales observadas en todas las variables analizadas apuntan a la necesidad de crear oportunidades educativas que favorezcan al desarrollo de destrezas intelectuales, de atención, de autoconcepto en general y de rendimiento académico, en particular de los niños ecuatorianos y especialmente de aquellos que presentan los niveles más bajos en las variables estudiadas puesto que podrían estar en riesgo de fracaso escolar. En suma, el conocimiento del comportamiento e interacción entre las variables personales y el rendimiento académico permitirán fundamentar el diseño e implementación de programas que ayuden a prevenir o mejorar el bajo rendimiento académico actual y en los años subsiguientes.

Una de las limitaciones del presente estudio es que se reportaron únicamente los puntajes globales de las variables personales, así como del rendimiento académico, lo cual no permitió un análisis más detallado de las habilidades cognitivas, atencionales, de autoconcepto, de ansiedad y desempeño escolar de los estudiantes. Por ello, futuras investigaciones deberían reportar las diferentes sub-habilidades dentro de cada variable, así como el rendimiento de los estudiantes en cada una de las áreas (i.e., matemáticas, lengua, ciencias naturales, ciencias sociales, entre otras). Otra limitación está relacionada con test de atención D2, empleado en este estudio, aun cuando es una de las pruebas más utilizadas para medir los niveles de atención de niños y jóvenes, sin embargo, no arroja una medida exacta de la capacidad atencional o de concentración, sino más bien una medida de la atención selectiva o capacidad de discriminación de estímulos. Pruebas más completas, y con mayor duración, como TOVA ${ }^{5}$ (Greenberg, 1996), serían más adecuadas para medir de los niveles atencionales de los estudiantes.

\section{REFERENCIAS}

Berg, D.H., 2008. Working memory and arithmetic calculation in children: The contributory roles of processing speed, short-term memory, and reading. Journal of Experimental Child Psychology, 99(4), 288-308.

Brickenkamp, R., 2002. D2, Test de atención. (Traducción al castellano por N. Seisdedos). Madrid, España: TEA Ediciones.

Castillo-Parra, G., E. Gómez, F. Ostrosky-Solís, 2009. Relación entre las funciones cognitivas y el nivel de rendimiento académico en niños. Revista Neuropsicología, Neuropsiquiatría y Neurociencias, 9(1), 41-54.

Cascón, I., 2000. Predictores del rendimiento académico en alumnos de primero y segundo de BUP. Disponible en http://www3.usal.es/ inico/investigacion/jornadas/ jornada2/comun/c19.html.

Caso-Niebla, J., L. Hernández-Guzmán, 2007. Variables que inciden en el rendimiento académico de adolescentes mexicanos. Revista Latinoamericana de Psicología, 39(3), 487-501.

Cullbertson, W., E.A. Zillmer, 1998. The tower of London (DX): A standardized approach to assessing executive functioning in children. Archives of Clinical Neuropsychology, 13(3), 285302.

Fandiño, Y.J., 2008. Enseñanza e investigación inteligentes de la inteligencia. Innovación Educativa, 8(44), $21 \mathrm{pp}$.

Fernández-Castillo, A., M.E. Gutiérrez Rojas, 2009. Atención selectiva, ansiedad, sintomatología depresiva y rendimiento académico en adolescentes. Electronic Journal of Research in Educational Psychology, 17(7), 49-76.

Gareca, S.B., 2005. Cultura, inteligencia y fracaso escolar: una tríada de complejo abordaje en la práctica docente. Revista Iberoamericana de Educación, 36(11), 12 pp.

5 TOVA: Test of variables of attention 
García-Sevilla, J., 1997. Psicología de la atención. Madrid, España: Síntesis.

García-Fernández, J., M.C. Martínez-Monteagudo, C.J. Inglés, 2013. ¿Cómo se relaciona la ansiedad escolar con el rendimiento académico? Revista Iberoamericana de Psicología y Salud, 4(1), 63-76.

García, T., C. Rodríguez, L. Betts, D. Areces, P. González-Castro, 2016. How affective-motivational variables and approaches to learning predict mathematics achievement in upper elementary levels. Learning and Individual Differences, 49, 25-31.

Geary, D.C., 1996. Sexual selection and sex differences in mathematical abilities. Behavioral and Brain Sciences, 19(2), 229-247.

Gonzalez-Pienda, J.A., 2003. El rendimiento escolar. Un análisis de las variables que lo condicionan. Revista Galego-Portuguesa de Psicoloxía e Educación, 8(7), 1138-1663.

Gottfredson, L.S., 2002. G: Highly general and highly practical. In: Sternberg, R.J., E.L. Grigorenko (Eds.). The general factor of intelligence. How general is it? Mahwah, NJ: Erlbaum, pp. 331338.

Greenberg, M.L., 1996. Test of variables of attention (TOVA). Los Alamitos, CA: Universal Attention Disorders.

Fernández-Castillo, A., M. Gutiérrez, 2009. Atención selectiva, ansiedad, sintomatología depresiva y rendimiento académico en adolescentes. Electronic Journal of Research in Educational Psychology, 7(17), 49-76.

Henández-Guamán, L., G. Bermúdez-Ornelas, S.H. Spence, M.J. Gonzales, J.I. Martínez-Guerrero, J. Aguilar, J. Gallegos, 2009. Versión es español de la Escala de Ansiedad para niños de Spence (SCAS). Revista latinoamericana de Psicología, 42(1), 13-24.

Hyde, J.S., M.C. Linn, 1988. Gender differences in verbal ability: A meta-analysis. Psychological Bulletin, 104(1), 53-69.

INEVAL, 2014. Ser estudiante 2013: Primeros resultados. Publicaciones INEVAL. Disponible en http://www.evaluacion.gob.ec/resultados/images/_in2_bin/DAGI_SEI2013 _InformeNacionalSE_20150701.pdf, 55 pp.

Jiménez, J.E., S. Hernández, E. García, A. Díaz, C. Rodríguez, R. Martín, 2015. Test de atención D2: Datos normativos y desarrollo evolutivo de la atención en educación primaria. European Journal of Education and Psychology, 5(1), 93-106.

Laidra, K., H. Pullmann, J. Allik, 2007. Personality and intelligence as predictors of academic achievement: A cross-sectional study from elementary to secondary school. Personality and Individual Differences, 42(3), 441-451.

Lambating, J., J.D. Allen, 2002. How the multiple functions of grades influence their validity and value as measures of academic achievement. Ponencia presentada en: Annual Meeting of the American Educational Research Association. New Orleans, April 1-5.

León, B., 2008. Atención plena y rendimiento académico en estudiantes de enseñanza secundaria. European Journal of Education and Psychology, 1(3), 17-26.

Locker, J., M. Cropley, 2004. Anxiety, depression and self-esteem in Secondary school children. School Psychology International, 25(3), 333-345.

Loperena, M.A., 2008. El autoconcepto en niños de cuatro a seis años. Tiempo de Educar, 9(18), 307-327.

Mazzone, L., F. Ducci, M.C. Scoto, E. Passaniti, V.G. D' Arrigo, B. Vitiello, 2007. The role of anxiety symptoms in school performance in a community sample of children and adolescents. $B M C$ Public Health, 7, 6 pp.

Milicic, M., 2001. Creo en ti: La construcción de la autoestima en el contexto escolar. Santiago, Chile: Lom Ediciones.

Miñano, P., J.L. Castejón, 2008. Capacidad predictiva de las variables cognitivo-motivacionales sobre el rendimiento académico. Revista Electrónica de Motivación y Emoción, 11(28), 13 pp. 
Ministerio de Educación, 2013. Archivo maestro de instituciones educativas AMIE. Reporte de registros educativos 2013-2014. Quito, Ecuador.

Naranjo, C.R., A.C. González, 2012. Autoestima en la adolescencia: análisis y estrategias de intervención. International Journal of Psychology and Psychological Therapy, 12(3), 389-403.

Pedhazur, E.J., 1997. Multiple regression in behavioral research: Explanation and prediction $\left(3^{\text {rd }}\right.$ ed.). Fort Worth, TX: Harcourt Brace College Publishers.

Piers, E.V., D.B. Harris, 1969. The Piers-Harris Children's Self-Concept Scale. Nashville, Tennesse: Counselor Recording and Tests.

Rains, D., 2004. Principios de neuropsicología humana. México D.F., México: McGraw-Hill Publ. Comp.

Sánchez, M., F. Peralta, 2003. Relaciones entre el autoconcepto y el rendimiento académico, en alumnos de Educación Primaria. Revista Electrónica de Investigación Psicoeducativa y Psicopedagógica, 1(1), 95-120.

Spinath, B., H.H. Freedenthaler, A.C. Neubauer, 2010. Domain-specific school achievement in boys and girls as predicted by intelligence, personality and motivation. Personality and Individual Differences, 48(4), 481-486.

Tejedor, F.J., S.G. González-Gonzales, M.M. García-Senoran, 2008. Estrategias atencionales y rendimiento académico en estudiantes de Secundaria. Revista Latinoamericana de Psicología, 40(1), 123-132.

Tenti Fanfani, E., 2002. El rendimiento escolar en la Argentina. Buenos Aires, Argentina: Losada.

TERCE, 2015. Logros de aprendizaje, Ecuador. Santiago, Chile: Oficina Regional de Educación de la UNESCO para América Latina y el Caribe. Disponible en http://www.unesco.org/new/fileadmin/ MULTIMEDIA/FIELD/ Santiago/pdf/Ficha-Logros-del-Aprendizaje-Ecuador.pdf.

TERCE, 2016. ¿Qué hay tras la inequidad de género en los logros de aprendizajes? Santiago, Chile: Oficina Regional de Educación de la UNESCO para América Latina y el Caribe. Disponible en http://unesdoc.unesco.org/images/0024/002442/244233s.pdf

Victor, M., A. Ropper, 2002. Principios de neurología. México D.F., México: McGraw-Hill Publ. Comp.

Villarroel, V.A., 2001. Relación entre autoconcepto y rendimiento académico. PSYKHE, 10(1), 3-18.

Wechsler, D., 2005. Wechsler Intelligence Scale for Children, Fourth Edition (WISC-IV), Spanish version. New York, NY: Psychological Corporation.

Yuste, C., M.A. Errisúriz, L. Ruiz, 2009. PIENSO Programa integral de estimulación de la inteligencia. México D.F., México: Trillas. 